\title{
Enseñanza del español, como segunda lengua en estudiantes indígenas, modalidad regular, URACCAN, Siuna, 2015
}

Jacoba Estela Rodríguez Andrade ${ }^{1}$ Julia del Carmen Argüello Mendieta ${ }^{2}$

\section{Resumen}

La investigación, se realizó con estudiantes indígenas del primer año de las carreras: Ingeniería Agroforestal, Administración de Empresas e Informática Administrativa de la Universidad de las Regiones Autónomas de la Costa Caribe Nicaragüense (URACCAN) Siuna. La finalidad fue analizar la situación de la enseñanza aprendizaje del español como segunda lengua, modalidad regular, describir las metodologías de enseñanza, estilos de aprendizaje, actitudes y compromisos que asumen los estudiantes. Es cualitativa y según el nivel de profundidad, descriptiva desde el enfoque del interaccionismo simbólico. Participaron once estudiantes indígenas, cinco mujeres, seis varones. Según su pertenencia étnica, siete miskitos, cuatro sumu-mayangnas. El universo y la muestra representan el cien por ciento. Las para la recolección de la información fueron: entrevistas, grupos focales, la observación y la revisión documental.

Los hallazgos, reflejan la variedad de metodologías que utilizan los docentes, en las que existe coincidencia sobre la metodología participativa, elaboración conjunta e interrogatorios. Los estilos de aprendizaje que se identificaron fueron: con mayor frecuencia el acomodador, en segundo lugar el divergente. Entre las estrategias propuestas para facilitar el proceso educativo del español como segunda lengua, se destacan: atención a la diversidad mediante tutorías de acompañamiento docente, la práctica de técnicas y hábitos de estudio.

Palabras clave: Metodologías de enseñanza; estilos de aprendizaje; estrategias de enseñanza.

\section{Summary}

The research was conducted with indigenous students of first year from the careers of: Agroforestry Engineering, Business Administration and Administrative Computing of the University of the Autonomous Regions of the Caribbean Coast of Nicaragua (URACCAN), from Siuna campus. The purpose was to analyze the situation

1 MSc. en Educación Intercultural Multilingüe. Asistente Bibliotecaria URACCAN, Las Minas. Docente Horario. estela46o@hotmail. com

2 MSc. en Metodología, Investigación y Evaluación de la Educación Superior. Coordinadora Autoevaluación Institucional URACCAN Las Minas. juliamendieta@yahoo.es 


\section{EDUCACIÓN}

of teaching-learning Spanish as a second language in the regular modality, describe the teaching methods, learning techniques, attitudes and commitments that are assumed by the students. The study is qualitative and according to the level of profoundness it's descriptive from the perspective of symbolic interactionism. Eleven indigenous students participated, five women, six men. According to their ethnicity seven were Miskitus and four Sumu-Mayangnas. The universe and the sample represents one hundred percent. The instruments for data collection that was used were based on interviews, focus groups, observation and document review.

The findings reflect the variety of methodologies that is used by the teachers, where there is agreement on the participative methodology, the joint elaboration as well as the interrogations. The learning techniques identified were: in first place the usher, and secondly the divergent. Among the strategies proposed to facilitate the educational process of Spanish as a second language we find: attention to diversity through teacher tutoring accompaniment, the practice of techniques and the implementation of study habits.

Keywords: Teaching methodologies; learning techniques; teaching strategies.

\section{Introducción}

En la actualidad, la Universidad de las Regiones Autónomas de la Costa Caribe Nicaragüense (URACCAN) no cuenta con investigaciones que evidencien como los docentes realizan la enseñanza del español en el desarrollo de sus clases y de qué forma los estudiantes indígenas, lo aprenden. Es por esa razón, que la investigación en el campo de la Educación Superior, específicamente en el contexto universitario, se desarrolló con el propósito de hacer un análisis de la situación de la enseñanza del español, como segunda lengua en el aprendizaje de los estudiantes indígenas.

Como aporte se espera que los resultados, sean insumos para la universidad y docentes que imparten la asignatura, para la toma de decisiones en relación a las posibles estrategias que faciliten el proceso enseñanza aprendizaje del español que les permita un mejor desenvolvimiento y comunicarse con éxito en ambientes interculturales.

El estudio que realizó en Chile, Abad y Toledo (2005), sobre factores claves en la enseñanza aprendizaje del español como lengua extranjera, expresan en los resultados, que los docentes deben hacer uso de metodologías didácticas adecuadas y considerar tres factores fundamentales: el énfasis en las competencias lingüísticas relacionadas con el nivel de lengua del estudiante; la integración de los contenidos de lengua y las habilidades comunicativas, finalmente, se recomienda atender las necesidades reales y específicas del estudiante, para lograr el objetivo de una comunicación precisa y efectiva por parte del doscente en el nuevo medio sociocultural que enfrenta (p.2). 
En Cuba el estudio de Escobedo Lora (2009), sobre la presencia del español en Canadá en el marco de la enseñanza de lenguas extranjeras, afirma que todo el sistema educativo canadiense, está tomando consciencia del interés de las personas, hoy en día por aprender español. En particular, los ministros de educación de algunas provincias, apoyan la enseñanza del español como lengua extranjera dentro del currículo de la enseñanza primaria y secundaria. Existe una creciente oferta del español en universidades, academias privadas, centros de educación para adultos y asociaciones españolas e hispanoamericanas (p.54).

En el Perú, el estudio de Arévalo, Pardo y Vigil (s.f), sobre la enseñanza del castellano como segunda lengua en las escuelas de Educación Intercultural Bilingüe (EBI) sostienen, que se deben introducir teorías de adquisición y técnicas de instrucción diferentes a las que guían la enseñanza del castellano como primera lengua. Los docentes deben ser capaces de desarrollar su trabajo de acuerdo a las necesidades y expectativas de sus estudiantes, es decir, tengan una competencia metodológica para la enseñanza del castellano como segunda lengua (p. 27).

\section{Revisión de literatura}

\section{Generalidades}

De acuerdo a Pozo (1999), citado por Martínez (2007), el concepto de enseñanza es:

Proceso mediante el cual el sujeto se forma gracias a actuaciones sociales y deliberadamente organizadas. Un proceso de formación que articula conocimientos, habilidades y actitudes, que se encaminan a la realización y mejora profesional o social, es decir al crecimiento personal.

La enseñanza nos remite más al quehacer didáctico, a la acción del docente. La enseñanza sólo podrá ser eficaz si parte de los conocimientos previos del estudiantado y logra activarlos y conectarlos adecuadamente con el material de aprendizaje"(Martínez, 2007).

Según Vigotsky citado por Rodríguez Ruiz y García-Merás García (s.f), el aprendizaje en el contexto escolar implica siempre adquisición de conocimiento y construcción de significado. De acuerdo con la tesis vigotskiana del aprendizaje el actor principal del proceso es el estudiante, aunque no es el único. El aprendizaje tiene lugar en un sistema interpersonal, por tanto a través de las interacciones con el docente y con los compañeros de aula, el estudiante aprende los instrumentos cognitivos y comunicativos de su cultura. El objetivo de la teoría de Vigotsky es descubrir y estimular la zona de desarrollo potencial o zona de desarrollo próximo en cada estudiante; en esta teoría se destaca la idea de que el sujeto no se limita a responder a los estímulos de modo pasivo o mecánico sino que actúa sobre ellos. 
Para Arríen (2000), es un privilegio docente orientar el aprendizaje en tres categorías principales: aprendizaje de saberes y su aplicación, habilidades y destrezas, valores y actitudes (p. 40). Un elemento esencial para un aprendizaje efectivo y de calidad es conocer a fondo la preferencia en la manera de aprender, la forma en que se absorbe y retiene la información. Dependiendo del estilo que se tenga, cada persona recogerá, organizará y transformará la información de forma distinta, por ello es muy importante identificar y examinar el estilo de aprendizaje, potenciar los comportamientos que resultan más efectivos y minimizar o evitar los que resultan adversos (Gispert, s.f, p. 33).

Según Moreno (2008), las metodologías constituyen un programa estratégico de la estructura técnica pedagógica del sistema educativo donde se pretende mejorar la educación. Estas surgen a partir de las confrontaciones de diversas experiencias concretas y la síntesis de los aportes de la teoría educativa.

\section{Metodologías de enseñanza}

En relación a las metodologías de enseñanza, Gamboa, Pérez, Zepeda y Rojas (1996) y Cid Lucero (2006), refieren que en la metodología activa participativa la estrategia de aprendizaje entendida como la forma en que los docentes orientan de manera dinámica y participativa, selecciona, organiza y desarrolla contenidos educativos, procedimientos, recursos y acciones que ocurren en los espacios educativos, con el propósito de cumplir los objetivos de aprendizaje. Se le denominan activas participativas porque se basan en experiencias concretas, dinámicas y proyectos de trabajos, donde el docente es facilitador (pp. 8-9).

Rosell Puig y Paneque Ramos (2009), refieren, que en el método de enseñanza de elaboración conjunta o dialogada, existe una participación activa a través de conversación entre docentes y estudiantes en la adquisición de conocimientos, mediante el papel dirigente del primero de forma inquisitiva, que exige una respuesta por parte de estudiantes. El contenido se va construyendo entre ambos en forma interactiva, propiciando la reflexión, el debate y la propuesta (p. 3).

Ajá Fernández et al., afirman: "la interrogación es un método didáctico verbal, el que resulta imprescindible en el desarrollo de la actividad docente", estimula el pensamiento, descubre cómo piensa o qué actitud manifiesta y comprueba y fomenta el conocimiento de aspectos nuevos (1997, p. 159).

Vargas Merina (2009), afirma que el método inductivo es cuando el asunto estudiado se presenta por medio de casos particulares... Genera gran actividad en los estudiantes, involucrándolos plenamente en su proceso de aprendizaje. El método deductivo es cuando el asunto que se estudia procede de lo general a lo particular. Presenta conceptos, principios, afirmaciones o definiciones (p. 5). 


\section{Estilos de aprendizaje}

Kolb (1999), describe los estilos de aprendizaje basados en la forma de procesar la información de la siguiente manera:

Estilo divergente: combina los pasos de aprendizaje de experiencia concreta y observación reflexiva. Las personas que adoptan este estilo de aprendizaje generalmente observan las situaciones concretas desde diferentes puntos de vista. El enfoque que utilizan es el de observador en vez de actuar.

Estilo asimilador: combina los pasos de aprendizaje de observación reflexiva y conceptualización abstracta. Las personas que adoptan este estilo de aprendizaje son generalmente las mejores para comprender una amplia variedad de información y ordenarla en forma concisa y lógica.

Estilo convergente: combina los pasos de aprendizaje de conceptualización abstracta y experimentación activa. Las personas que se identifican con este estilo de aprendizaje suelen ser las mejores para descubrir la aplicación práctica de las ideas y teorías.

Estilo acomodador: combina los pasos de aprendizaje de experimentación activa y experiencia concreta. Las personas que adoptan este estilo de aprendizaje tienen la capacidad de aprender principalmente de la experiencia "concreta". Si este es su estilo, es probable que disfrute ejecutando planes y participando en experiencias nuevas y que presenten desafíos (p. 145).

\section{Estrategias en la enseñanza del español como segunda lengua del estudiantado indígena}

Estrategias propuestas a docentes que imparten la asignatura de Idioma Español a estudiantes indígenas:

En la universidad, se debe efectivizar la atención a la diversidad entendida como el conjunto de acciones educativas que intentan dar respuesta a las necesidades, intereses, motivaciones y capacidades de todos los estudiantes, con la finalidad de que puedan alcanzar el máximo desarrollo posible de sus capacidades personales, los objetivos establecidos y las competencias básicas (Dubón V, 2010).

Sandoval y Guerra (2007), señalan que la tutoría en las Universidades Interculturales de Educación Superior, se entiende como un rol y proceso de ayuda que utiliza modalidades y define metas en relación con los valores culturales de los tutorados, reconoce su identidad y acepta al grupo en una dimensión más amplia; avoca el uso de estrategias específicas y roles en los procesos culturales y trata de equilibrar la 
importancia del individualismo y el colectivismo en el establecimiento, diagnóstico y tratamiento del tutorado.

A los estudiantes indígenas (Mujeres y hombres) se proponen practicar hábitos de estudio como: la lectura, visitas a biblioteca para la utilización de libros y otros materiales, el tiempo, el lugar de estudio y la planificación, los que según Vinet (2006) citado por Cruz (2011), deben ser entendidos como la continua repetición de un acto, que hace posible lograr resultados positivos en el aprendizaje y donde intervienen factores como el interés y la motivación interna del estudiante que aprende.

Técnicas de estudio: Asistencia a clase acompañada de una buena atención a las explicaciones, la técnica de lectura, del subrayado, toma de apuntes, la concentración, la investigación y la técnica de analizar. En relación con las técnicas de estudio, León Gross (2004) y Hernández (2005), citado por Escalante, Linzaga, Merlos (2008), las define como ayudas prácticas para la tarea de estudiar con actividades visibles y operativas que facilitan la adquisición de conocimientos.

\section{Materiales y métodos}

La investigación se realizó en la Universidad de las Regiones Autónomas de la Costa Caribe Nicaragüense (URACCAN) Siuna, ubicada en el barrio Pedro Joaquín Chamorro, sector 3, en el 2015. Es cualitativa y según el nivel de profundidad es descriptiva y se enmarca bajo el paradigma del enfoque teórico metodológico del interaccionismo simbólico.

La población, fueron once estudiantes indígenas de primer año de la modalidad regular, cinco mujeres y seis varones. Según su pertenencia étnica, siete miskitos y cuatro sumu-mayangnas de Ingeniería Agroforestal, Administración de Empresas e Informática Administrativa. Para la obtención de la información se utilizaron: Fuentes primarias: Estudiantes indígenas de primer año de la modalidad regular y docentes que imparten clases en la asignatura de Idioma Español. Fuentes secundarias: literatura física y digital que ofrece el internet, registro académico, programas de español, investigaciones monográficas que se relacionan con el tema de investigación. Mediante las técnicas: entrevistas, grupos focales, observación y la revisión documental.

El procesamiento consistió en la revisión a detalle de la información y se ordenó por técnicas, preguntas, descriptores y se clasificó de acuerdo a los objetivos de la investigación. Se hizo una transcripción literal de las respuestas que brindaron los informantes.

El análisis de la información, se realizó mediante la organización y reducción de los datos según los descriptores de análisis. Se procedió a una descripción analítica de los relatos escritos y grabados en los grupos focales, entrevistas y observaciones. 
A la vez, se realizó la discusión y comparación de los hechos y expresiones de los informantes con los postulados teóricos de diferentes autores.

\section{Resultados y discusión}

\section{Metodologías utilizadas por los docentes de español con estudiantes indígenas, modalidad regular de la URACCAN Siuna, 2015}

En las entrevistas realizadas a docentes de español, expresaron que por excelencia utilizan con estudiantes indígenas la metodología activa participativa, porque se les facilita enseñarles, crearles y desarrollar habilidades y competencias en sus aprendientes para hablar en público, en grupo, de manera espontánea o libre. Lo anterior se asemeja con Gamboa, Pérez, Zepeda y Rojas (1996), Cid Lucero (2006), quiénes refieren que en la metodología activa la estrategia de aprendizaje es entendida como la forma en que se orienta de manera dinámica y participativa, la selección, organización y desarrollo de los contenidos educativos para cumplir los objetivos de aprendizaje. Son activas participativas basadas en las experiencias concretas, dinámicas y proyectos de trabajos.

Se constató que los docentes utilizan la metodología activa participativa, hacen la clase dinámica, participativa y organizada con la finalidad de cumplir el objetivo propuesto, sin embargo la participación de los estudiantes indígenas es muy limitada.

Otro método para impartir la asignatura de Idioma Español, es el de elaboración conjunta, que se utiliza para establecer una conversación frecuente que permite que los estudiantes participen y aporten información de un determinado contenido, en el que docente y estudiante construyen conceptos y definiciones. Lo antes descrito, se relaciona con Rosell Puig y Paneque Ramos (2009), que refieren que en el método de enseñanza de elaboración conjunta o dialogada... hay una participación activa a través de conversación o diálogo... entre docente y estudiante en la adquisición de conocimientos. El contenido se construye entre ambos en forma interactiva, propicia la reflexión y el debate.

En la observación, se constató que los docentes utilizan el método de elaboración conjunta. Los estudiantes, presentaron sus trabajos prácticos de forma individual, los que fueron corregidos y mejorados en conjunto. Incluso se surgieron nuevas ideas, aclararon dudas, pero la participación de estudiantes indígenas fue poca.

A través de la entrevista, se encontró que otro método que utilizan los docentes en la enseñanza del español con estudiantes indígenas, es el interrogatorio que se hace a través de una discusión grupal con preguntas individuales directas. Los docentes guían los aportes y ayudan con sus observaciones para aclarar dudas, de tal manera que la información sea más completa según el objetivo que se persigue. Lo que coincide 


\section{EDUCACIÓN}

con Ajá Fernández et al. (1997), que expone "la interrogación es un método didáctico verbal, el que resulta imprescindible en el desarrollo de la actividad docente".

Se observó que se utiliza el método interrogatorio de forma individual y grupal al presentar los trabajos realizados, donde los docentes dirigieron el proceso a través de preguntas y estudiantes defendieron sus criterios y aclararon dudas del contenido; sin embargo, los estudiantes indígenas participan muy poco en comparación con los estudiantes mestizos.

En la entrevista que se aplicó a los docente de la carrera de Informática Administrativa, opinó que otro método que utiliza en la asignatura de español es el inductivo, el pensamiento va de lo particular a lo general, de lo conocido a lo desconocido. Utiliza impulsos para llegar a definiciones y conceptos al tratar nuevos contenidos, parte de la experiencia que poseen sus estudiantes. Lo que refuerza Vargas Merina (2009), al afirmar que el método inductivo es cuando el asunto estudiado se presenta por medio de casos particulares. Este método genera gran actividad en los estudiantes, los involucra plenamente en su proceso de aprendizaje.

De igual manera, el docente expresó que en el método deductivo, el pensamiento va de lo general a lo particular, donde se elaboraron conceptos y definiciones para extraer conclusiones y consecuencias de un determinado contenido. Lo que coincide con lo que afirma Vargas Merina (2009), quien refiere que el método deductivo es cuando el asunto que se estudia procede de lo general a lo particular. Los docentes presentan conceptos, principios, afirmaciones o definiciones de las que se extraen conclusiones y consecuencias.

Las metodologías que utilizan son modernas y menos tradicionalistas. Buscan la participación activa de los estudiantes. Se trata de seguir procesos de pensamiento crítico, creativo, reflexivo y de análisis. Se procura establecer vínculos próximos a la experiencia, el trabajo y la práctica de lo teórico de manera individual o grupal. Se aprovechan estas situaciones para formar el respeto mutuo y demás valores, así como la motivación y la participación en sus propios procesos para aprender, desarrollar habilidades y competencias lingüísticas que les permita una comunicación exitosa en los entornos en los que se desenvuelven.

En la revisión documental, se constató a través de la lectura de los programas de la asignatura de Idioma Español de las tres carreras en relación con las orientaciones metodológicas, que no establece ninguna diferencia en la enseñanza del mismo, dirigidos a estudiantes indígenas, carecen de una orientación de cómo se debe seleccionar el método de enseñanza tomando en consideración las características actitudinales y de comportamiento de sus grupos y de acuerdo con los contenidos a desarrollar y no precisamente para dar respuesta a las necesidades de formación y desarrollo de competencias y habilidades comunicativas de sus estudiantes indígenas en específico. 
La universidad, tendría que incluir en las readecuaciones curriculares desde los objetivos y orientaciones metodológicas de la asignatura de Idioma Español, cómo se debe atender a estudiantes indígenas. También, es necesario que los docentes en la enseñanza del español, estén conscientes de las diferencias entre estudiantes indígenas y mestizos, que les permita promover actividades de aprendizaje integradores, relaciones recíprocas efectivas, brindar confianza al estudiantado indígena para participar y comprender que sus aportes se relacionan con los significados que implican la vida comunitaria y el mundo que les rodea.

\section{Estilos de aprendizaje utilizados con estudiantes indígenas e}

En el grupo focal que se aplicó a estudiantes indígenas se pudo constatar que una minoría se encuentra en un estilo divergente. Expresaron que al realizar los trabajos en grupos tienen espacios de participación y se logra llegar a un consenso después de debatir para entregar el trabajo, de manera que se visualiza una práctica cultural de intercambio de conocimientos, que se manifiesta en una experiencia como elemento significativo en la construcción del conocimiento. Lo que se fundamenta en lo planteado por Kolb (1999), expresa que el estilo de aprendizaje divergente es donde él o la aprendiente combina los pasos de aprendizaje de experiencia concreta y observación reflexiva y prefieren trabajar en grupos para recopilar información, escuchar con una mente abierta y recibir realimentación personalizada.

Lo anterior, refleja la intencionalidad de los estudiantes indígenas al interactuar y establecer relaciones para adquirir y construir el conocimiento, lo que se evidencia en un aprendizaje de interacción de pensamiento. Otro estilo de aprendizaje que se encontró en el grupo focal con estudiantes indígenas, es el acomodador el que se manifiesta en la capacidad de aprender de la experiencia y se relaciona con las situaciones de aprendizaje que vinculan el trabajo en grupo. Este estilo, es el más utilizado por la mayoría de aprendientes indígenas.

Es importante señalar que este tipo de estudiante, depende mucho del trabajo que realicen sus colegas, a pesar de interactuar en grupos, el comportamiento es pasivo y se enmarca en un aprendizaje de experiencia concreta y experimentación activa, pero un elemento significativo y que acompaña este comportamiento, es que el confía en información que le brindan las personas.

El estudiantado miskitu y mayangna considera que su aprendizaje es mayor cuando los docentes dirigen la clase. Manifestaron, que se sienten satisfechos cuando estos les hacen preguntas y cada uno brinda sus aportes e ideas y así aprenden. Se sienten conformes cuando los docentes les proporcionan y asumen que este debe facilitar y conducir su aprendizaje. Asimismo, confían en sus colegas mestizos. 


\section{EDUCACIÓN}

Los docentes en sus entrevistas manifestaron que los estudiantes indígenas se encuentran en un estilo acomodador, porque desde los estudios de Educación Secundaria el sistema educativo los acomodó a ser meramente reproductivos, lo que dificulta llegar a un proceso de aprendizaje productivo, a ser partícipes de su propio aprendizaje. Los estudiantes confían plenamente en lo que enseña él o la docente y se conforman demasiado con lo que se les brinda.

Situación que respalda Kolb (1999), al mencionar que los estudiantes que se inclinan más hacia este estilo de aprendizaje tienen la capacidad de aprender principalmente de experiencias concretas en vez de analizar lógicamente una situación... Confían en información que le brindan sus docentes, en vez de basarse en su propio análisis técnico. Es posible que prefiera trabajar junto con otras personas para lograr que los objetivos se cumplan, hacer labores de campo y comprobar diferentes enfoques para completar un proyecto.

Se corroboró mediante la observación, que para el estudiantado indígena les es satisfactorio trabajar en grupos, se sienten a gusto y participan en la realización del trabajo. Además, hacen uso de la escucha de otras experiencias y logran que sea efectivo mediante la observación detallada de cada proceso de aprendizaje y sus aportes los relacionan con la información que poseen de la vida cotidiana comunitaria de sus lugares de procedencia.

Se deduce que de los cuatro estilos propuestos por Kolb, los estudiantes indígenas de la URACCAN - Siuna Las Minas, hacen uso de dos: el divergente y el acomodador. Se evidencia que en ambos estilos para el estudiantado indígena, tiene un significado especial las discusiones grupales que se dan para enriquecer el conocimiento y se considera que los mismos son producto de las interacciones entre estudiantes y docentes.

Los resultados demuestran, que ocho estudiantes indígenas con estilo acomodador se encuentran en procesos de aprendizaje que les permite fortalecer su capacidad de análisis, reflexión, crítica y construir sus propios conocimientos y los tres estudiantes indígenas que practican el estilo divergente al estudiar subrayan, leen y toman notas y la forma en la que procesan la información tiene relación con el estilo de aprendizaje que describe Kolb.

En coherencia con lo que se abordó anteriormente, un elemento esencial en el aprendizaje efectivo para el estudiantado indígena es conocer la preferencia en la manera de aprender, la forma en que se selecciona y retiene la información. De acuerdo al estilo que se tenga, cada persona recopila, organiza y transforma la información de forma distinta; pero, es importante identificar y examinar el estilo de aprendizaje, potenciar los comportamientos que resultan más efectivos y minimizar o evitar los que resultan adversos. 


\section{Estrategias en Idioma Español como segunda lengua en estudiantes indígenas}

Estos resultados, proporcionan elementos para algunas estrategias prácticas para los docentes de la universidad, que permitan fortalecer el español como segunda lengua, además plantear estrategias a estudiantes indígenas para el logro de aprendizajes significativos.

Abad y Toledo (2005), señalan fundamental que los docentes conozcan el nivel de lengua del hablante, las cuales según las directivas de ACTFL (Proficiency Guidelines, American Council on the Teaching of Foreign Languages), citado por Swender (1999), señalan cuatro grandes niveles (principiante, intermedio, avanzado y superior).

A partir de ello, se propone que para atender al estudiantado indígena en la universidad se debe efectivizar la atención a la diversidad entendida como el conjunto de acciones educativas que intentan dar respuesta a las necesidades, intereses, motivaciones y capacidades de todos los estudiantes, con la finalidad de que puedan alcanzar, el máximo desarrollo posible de sus capacidades personales, los objetivos que se establecen y las competencias básicas (Dubón V., 2010).

Lo que se sustenta en lo que refieren Sandoval y Guerra (2007), señalan que la tutoría en las Universidades Interculturales de Educación Superior se entiende como un rol y proceso de ayuda que utiliza modalidades y define metas en relación con los valores culturales de los tutorados, reconoce su identidad y acepta al grupo en una dimensión más amplia; apunta al uso de estrategias específicas y roles en los procesos culturales y trata de equilibrar la importancia del individualismo y el colectivismo en el establecimiento, diagnóstico y tratamiento del tutorado.

Las tutorías constituyen el espacio ideal para la promoción de la interculturalidad como el eje estratégico para hacer visible la diversidad étnica de los estudiantes y desarrollar acciones para atender las problemáticas de la población estudiantil indígena y potenciar sus capacidades y habilidades, a través del desarrollo de programas de acompañamiento y políticas compensatorias que coadyuven a una experiencia escolar positiva.

Se considera vital, utilizar estrategias de aprendizaje para el dominio del español como segunda lengua en estudiantes indígenas, a través de la práctica de hábitos y técnicas de estudio, que se utilicen no sólo en esta asignatura, sino en el transcurso de la carrera universitaria.

A los estudiantes indígenas se propone practicar: el hábito de la lectura, visitas a biblioteca para la utilización de libros y otros materiales, el tiempo, el lugar de estudio y la planificación, los que según Vinet (2006), citado por Cruz (2011), se deben entender 
como la continua repetición de un acto, que hace posible lograr resultados positivos en el aprendizaje y donde intervienen factores como el interés y la motivación interna del estudiante que aprende.

Se sugieren las técnicas de estudio: asistencia a clase, técnica de lectura, del subrayado, toma de apuntes, la concentración, la investigación y la técnica de analizar, definidas por León Gross (2004) y Hernández (2005), citado por Escalante, Linzaga, Merlos (2008), como ayudas prácticas para la tarea de estudiar con actividades visibles y operativas que facilitan la adquisición de conocimientos.

\section{Conclusiones}

Las metodologías de enseñanza utilizadas por los docentes de la asignatura de español con estudiantes indígenas son variadas y existe coincidencia en la aplicación de la activa participación, elaboración conjunta y el interrogatorio.

Los estilos de aprendizaje identificados en los estudiantes indígenas en la asignatura de español fueron: divergente y acomodador, reflejándose con mayor frecuencia el acomodador y en segundo lugar el divergente.

Las estrategias propuestas para facilitar el proceso de enseñanza aprendizaje del español como segunda lengua son la atención a la diversidad a través de tutorías de acompañamiento a estudiantes indígenas por docentes y práctica de hábitos y técnicas de estudio en el caso del estudiantado indígena.

\section{Recomendaciones}

\section{A la URACCAN}

Planificar mecanismos que coadyuven la atención al estudiantado indígena, mismos que pueden consistir en cambios o reformas curriculares y programas de atención a las trayectorias escolares. Ofrecer tutorías de acompañamiento individual o grupal, acorde a las necesidades de formación en los estudiantes indígenas incluidos desde los programas de asignatura de Idioma Español en las carreras de la modalidad regular.

Realizar una revisión de los programas de las carreras de Ingeniería Agroforestal, Administración de Empresas e Informática Administrativa, ya que carecen de orientaciones metodológicas, sobre cómo brindar atención a estudiantes indígenas en la asignatura de Idioma Español. Incorporar en los programas de esta asignatura en los objetivos y metodologías la atención a estudiantes indígenas. 


\section{A docentes que imparten la asignatura de Idioma Español}

Brindar una atención diferenciada a los estudiantes indígenas que presentan mayor dificultad de aprendizaje y especialmente en la asignatura de Idioma Español. Propiciar actividades de trabajo en las aulas de clases que permitan la integración de estudiantes indígenas con los mestizos y afrodescendientes que contribuya a eliminar el rechazo o actitudes discriminatorias entre el estudiantado.

Realizar un diagnóstico en el estudiantado indígena de primer año en la asignatura de Idioma Español, para identificar los estilos de aprendizaje de los aprendientes y ofrecer una enseñanza pertinente a sus necesidades de formación. Desarrollar círculos de estudio con estudiantes indígenas monitores para apoyarse con mayor dificultad en la asignatura de Idioma Español.

\section{A la coordinación de la carrera en la URACCAN}

Revisar y validar las estrategias propuestas con docentes de español con el objetivo de hacerlas efectivas en el entorno educativo para facilitar el proceso de enseñanza aprendizaje del español como segunda lengua de los estudiantes indígenas.

Realizar una revisión de los programas de las carreras de Ingeniería Agroforestal, Administración de Empresas e Informática Administrativa, ya que carecen de orientaciones metodológicas sobre cómo brindar atención a estudiantes indígenas en la asignatura de Idioma Español.

\section{A estudiantes indígenas:}

Asuman su compromiso y responsabilidad con su aprendizaje, especialmente en esta asignatura de Idioma Español que fortalezca sus habilidades comunicativas tanto oral como escrita. Tomen consciencia de la importancia que tiene la adquisición y aplicación de hábitos y técnicas de estudio para lograr aprendizajes significativos de acuerdo con sus habilidades, especialmente en esta asignatura.

\section{Lista de referencias}

Abad, M., \& Toledo, G. (2005). Factores claves en la enseñanza- aprendizaje del español como lengua extranjera. Chile: Pontificia Universidad Católica de Chile.

Ajá Fernández, J. M., Albaladejo Marcet, C., Balada Monclús, M., Banda Tarradellas, A., Bartolomé Pina, M., Benedito Antolí, V., et al. (1997). Manual de la educación. Barcelona, España: Océano. 


\section{EDUCACIÓN}

Arévalo, I., Pardo, K., \& Vigil, N. (s.f). Enseñanza de castellano como segunda lengua en las escuelas EBI del Perú. Perú: Dirección Nacional de Educación Bilingüe Intercultural.

Arríen, J. (2000). Motivación y aprendizaje en el aula de clase. Madrid, España: Santillana.

Cid Lucero, M. (2006). Didáctica de la lengua miskitu, material didáctico para la formación docente. Bilwi, Puerto Cabezas, Región Autónoma Atlántico Norte: Ford Foundation.

Cruz, F. (2011). Hábitos de estudio, actitudes y autoestima relacionados con rendimiento académico en estudiantes de enfermería. Cuaderno de Educación y Desarrollo. Revista académica semestral, 3 (23). Recuperado de: http://www.eumed.net/ $\mathrm{rev} / \mathrm{ced} / 23 / \mathrm{fcn} . \mathrm{htm}$

Dubón V., M. (2010). Metodología didáctica. Siuna, Nicaragua: URACCAN.

Escalante, L., Linzaga, C., \& Merlos, M. (2008). Comportamiento de estudiantes en función a sus hábitos de estudio. Revista Actualidades de los Investigativas en Educación. 8 (2). Recuperado de: http://revista.inie.ucr.ac.cr/ediciones/controlador/Article/ accion/show/articulo/comportamiento de los estudiantes en función a sus hábitos de estudio.html.

Escobedo Lora, D. (2009). La presencia del español en Canadá en el marco de la enseñanza de lenguas extranjeras. Santiago de Cuba: Universidad de Oriente.

Gamboa, A., Pérez, S., Zepeda, Y., \& Rojas, M. (1996). Manual para el trabajo con los padres y madres de familia de preescolar. Managua, Nicaragua: Ministerio de Educación.

Gispert, C. (s.f). Enciclopedia de la psicopedagogía: psicología y educación, Vol. 1. España: Océano.

Kolb, D. (1999). Inventarios de estilos de aprendizajes. Guatemala: Educación de adultos.

León Gross, E. (2004). Técnicas de estudio: claves para mejorar el rendimiento intelectual. España: LIBSA.

Martínez, P. (2007). Orientaciones didácticas para trabajar los estilos de aprendizaje en el aula (Educación Secundaria) en II Congreso Internacional de estilos de aprendizaje. Chile: Universidad de Concepción.

Moreno, M. (2008). Las metodologías didácticas y los recursos didácticos. Costa Rica: UNED. 
Rodríguez Ruiz, M., \& García-Merás García, E. (s.f.). Las estrategias de aprendizaje y sus particularidades en lenguas extranjeras. Revista Iberoamericana de Educación. Recuperado de: http://www.rieoei.org/deloslectores/965Rodriguez.PDF

Rosell Puig, W., \& Paneque Ramos, E. R. (2009). Consideraciones generales de los métodos de enseñanza y su aplicación en cada etapa del aprendizaje. Revista Habanera de Ciencias Médicas versión On-line. Recuperado de: http://scielo.sld.cu/scielo. php?script=sci_arttext\&pid=S1729-519X2009000200016

Sandoval, A., \& Guerra, E. (2007). La interculturalidad en la Educación Superior en México. México: Universidad Autónoma Indígena de México.

Swender, E. (Ed.). (1999). Oral Proficiency Interview Tester Training Manual. Chile: ACTFL Inc.

Vargas Merina, A. (2009). Métodos de enseñanza. Granada, España: McGraw-Hill. 